\title{
Los clasificadores de posesión en wichi
}

\author{
Possessive classifiers in the Wichí language \\ Os classificadores de posse em wichí
}

\author{
Lorena Cayré Baito \\ Universidad Nacional del Nordeste - Argentina
}

\begin{abstract}
The aim of this paper is to analyze the possessive classifiers $k a$, lo and $k o$ of the Wichí language. In the Wichí reviewed literature, only $k a$ and $l o$ have been described and studied. This article describes, compares and analyzes the morphosyntatic characteristics, compatibilities and notions of possession of the three classifiers according to Heine's (1997) approach. The following hypothesis is proposed here: a possession scale in which, on the one hand, possessive constructions with $k o$ and $l o$ express permanent possession (in the sense that the possessor it is always, in some way, related to the possessed item; therefore it is possible to affirm that such an item belongs to that possessor). On the other hand, possessive constructions with $k a$ express temporal and/or physical possession as well as permanent possession; but in this case, the possessed item may belong to the possessor or not.
\end{abstract}

\section{Keywords}

Possessive classifiers, Wichí, Notions of possession. 


\section{Resumo}

O objetivo deste artigo é analisar os classificadores de posse $k a$, lo e ko da língua wichí. Na bibliografia wichí consultada, somente $k a$ e lo têm sido descritos e estudados. Neste artigo, são descritas, comparadas e analisadas as características morfossintáticas, compatibilidades e noções de posse dos três classificadores de acordo com o enfoque teórico de Heine (1997). Na análise propõe-se, como hipótese, uma escala de posse em que, por um lado, as construções possessivas com ko e lo expressam noções de posse permanente (no sentido de que o possuidor sempre está relacionado, de alguma forma, com o item possuído, sendo possível, portanto afirmar que o item possuído lhe pertence). Por outro lado, as construções possessivas com $k a$ expressam noções de posse temporal e/ou física e, também, noções de posse permanente mas, nesse caso, o item possuído pode pertencer ou não ao possuidor.

\section{Palavras-chave}

Classificadores de posse, Wichí, Noções de posse. 


\section{Introducción}

7 l wichí presenta dos clasificadores de posesión, ka y lo, que son usados

1 en construcciones posesivas sólo con nombres alienables. De acuerdo

1 con la bibliografía wichí consultada, $k a$ es analizado como un clasificador general que categoriza a todos los nombres alienables y lo como un clasificador específico de uso exclusivo para nombres referidos a animales (GOLLUSCIO, 1993; TERRAZA, 2009).

Además de estos dos clasificadores hemos identificado la presencia de otro clasificador, $k o$, que a diferencia de $k a$ y lo puede ser usado en construcciones posesivas con nombres inalienables. En este trabajo nos proponemos estudiar los clasificadores de posesión observados en nuestro corpus mediante el análisis de sus particularidades, compatibilidades y diferencias semánticas (esto es en qué tipo de construcciones posesivas son usados los clasificadores y qué nociones de posesión expresan).*

Enmarcamos nuestro análisis tomando como referencia los planteamientos teóricos de Aikhenvald (2003), Carlson y Payne (1989) y Heine (1997).

El corpus que utilizamos para realizar este trabajo está compuesto de paradigmas y oraciones breves de un hablante wichí, SM, ${ }^{1}$ oriundo de Ingeniero Juárez, provincia de Formosa, quien reside actualmente en la ciudad de Resistencia (Chaco). ${ }^{2}$

\section{Marco teórico}

Organizamos el marco teórico en dos partes, en la primera parte desarrollamos el concepto de clasificadores, principalmente, los clasificadores de posesión. En la segunda, presentamos el concepto de posesión desarrollado por Heine (1997). 


\subsection{Los clasificadores de posesión}

De acuerdo con Aikhenvald (2003) los clasificadores son morfemas que denotan ciertas características sobresalientes de una entidad a la cual un nombre se refiere. Los tipos de clasificadores que pueden encontrarse en las lenguas son: clasificadores nominales, clasificadores numerales, clasificadores posesivos, clasificadores verbales, clasificadores locativos y clasificadores deícticos.

\subsubsection{Los clasificadores en construcciones posesivas}

Aikhenvald distingue tres formas de categorizar nombres en una construcción posesiva que se corresponden con tres tipos de clasificadores: clasificadores del nombre poseído, clasificadores relacionales y clasificadores del nombre poseedor.

Estos clasificadores presentan las siguientes características generales:

- Morfológicamente pueden ser palabras independientes o afijos.

- No se expresan fuera de la frase nominal posesiva.

i. Clasificadores del nombre poseído

Categorizan al nombre poseído en términos de animacidad, forma, función y consistencia. No obstante, Aikhenvald señala que algunas lenguas presentan un clasificador 'general' que reemplaza otros clasificadores más específicos. Por ejemplo, la lengua yavapai (lengua yuman) tiene dos clasificadores, un clasificador específico para categorizar nombres referidos a mascotas y animales domésticos y otro clasificador más general (AIKHENVALD, 2003, p. 127).

Los clasificadores del nombre poseído no dependen necesariamente de la distinción entre posesión alienable y posesión inalienable, por ello es posible identificar dos tipos de clasificadores: clasificadores usados sólo con una subclase de nombres alienables ${ }^{3}$ y clasificadores usados independientemente del tipo de posesión alienable e inalienable (tanto en lenguas que presentan esta distinción gramatical como en lenguas que no presentan tal distinción). ${ }^{4}$

ii. Clasificadores del nombre poseedor

Categorizan al poseedor en una construcción posesiva pero son extremadamente raros en las lenguas del mundo. La elección del clasificador está determinada por las propiedades del poseedor: animado/no animado ${ }^{5} \mathrm{y}$ humano/ no humano. 
iii. Clasificadores relacionales

Los clasificadores relacionales a diferencia de los anteriores, categorizan la naturaleza semántica de la relación entre el poseedor y el poseído. La elección de la marca posesiva está determinada por la forma en que el poseedor se relaciona con el poseído.

Carlson y Payne (1989) sostienen que el sistema de clasificadores genitivos, a diferencia de otros sistemas (clasificadores nominales, numerales y verbales), está fundamentado en categorías funcionales más que en categorías físicas. Las posibles interacciones o asociaciones que pueden establecerse con unítem se basan en la función ${ }^{6}$ o utilidad de éste más que en su forma física; es decir, para qué es bueno un determinado ítem y para qué sirve. De este manera, los clasificadores genitivos no expresan solamente una relación de posesión, sino que indican o codifican numerosos tipos de relaciones o asociaciones entre el poseedor y el poseído. Estas asociaciones o relaciones individualizadas ${ }^{7}$ pueden incluir: relaciones de parentesco: mi mamá; roles culturales: mi maestra; lugar de origen y residencia: mi país, mi ciudad, mi barrio; propiedad individualizada genuina: $\mathrm{mi}$ machete; ítems para beneficio propio -aunque no sean claramente poseídos-: mi dinero, mi universidad, mi oficina; medio de transporte: mi tren sale a las 5 y características abstractas: mi estilo (CARLSON; PAYNE, 1989, p. 110).

Los clasificadores relacionales casi siempre están limitados a construcciones de posesión alienables. De acuerdo con Aikhenvald la forma en que las relaciones posesivas pueden categorizarse mediante los clasificadores relacionales, presenta similitudes con la distinción gramatical entre posesión alienable e inalienable:

- Todas las lenguas con clasificadores relacionales distinguen posesión alienable y posesión inalienable. Los clasificadores de posesión están limitados a los nombres poseídos alienables.

- En lenguas con distinción gramatical entre posesión alienable e inalienable, algunos nombres pueden ser tratados como alienables o inalienables. Los clasificadores relacionales en estas lenguas son usados de igual manera que la distinción inalienable/alienable, indicando la forma en que los nombres son poseídos. ${ }^{8}$

Las diferencias entre estos tres tipos de clasificadores pueden sistematizarse de la siguiente manera: 


\section{CUADRO 1}

Diferencias entre los clasificadores de posesión (Basado en AIKHENVALD, 2003, p. 145-146)

\begin{tabular}{l|l|l|l}
\hline & $\begin{array}{l}\text { Clasificadores del } \\
\text { nombre poseedor }\end{array}$ & $\begin{array}{l}\text { Clasificadores } \\
\text { relacionales }\end{array}$ & $\begin{array}{l}\text { Clasificadores del } \\
\text { nombre poseído }\end{array}$ \\
\hline $\begin{array}{l}\text { Componente } \\
\text { categorizado en la } \\
\text { construcción posesiva }\end{array}$ & $\begin{array}{l}\text { Nombre poseedor } \\
\text { (categorizan a un } \\
\text { nombre) }\end{array}$ & $\begin{array}{l}\text { Tipo de relación } \\
\text { posesiva (categorizan } \\
\text { una relación de posesión) }\end{array}$ & $\begin{array}{l}\text { Nombre poseído } \\
\text { (categorizan a un } \\
\text { nombre) }\end{array}$ \\
\hline $\begin{array}{l}\text { Semántica } \\
\text { Animado/no animado; } \\
\text { humano/no humano }\end{array}$ & $\begin{array}{l}\text { La forma en que un } \\
\text { nombre puede ser poseído }\end{array}$ & $\begin{array}{l}\text { Animacidad, } \\
\text { tamaño, forma, etc. }\end{array}$ \\
\hline $\begin{array}{l}\text { Relación con posesión } \\
\text { alienable/inalienable }\end{array}$ & $\begin{array}{l}\text { No desarrollado } \\
\text { por la autora }\end{array}$ & $\begin{array}{l}\text { Usados sólo con } \\
\text { posesión alienable }\end{array}$ & $\begin{array}{l}\text { Usados indepen- } \\
\text { dientemente del } \\
\text { tipo de posesión }\end{array}$ \\
\hline $\begin{array}{l}\text { Frecuencia en las } \\
\text { lenguas }\end{array}$ & Rara & Media & Común \\
\hline Tamaño del inventario & Pequeño & Pequeño & Puede ser grande \\
\hline
\end{tabular}

A pesar de estas diferencias Aikhenvald señala que los clasificadores del nombre poseído y los clasificadores relacionales pueden combinarse e integrar un sistema, que marca tanto la forma en que un ítem puede ser poseído como las características del ítem en sí mismo. Por ejemplo, en la lengua truquese (lengua austronesia) el clasificador wúnúma 'potencialmente bebible' es usado no sólo para categorizar al nombre kkóniki 'agua’ como bebible, sino también para indicar la forma en que éste es manipulado o manejado: ítem que pude ser consumido, bebido (AIKHENVALD, 2003, p. 141-142).

También es posible (aunque tipológicamente es muy raro) encontrar lenguas en las que los clasificadores relacionales y los clasificadores del nombre poseído formen un sistema separado e independiente en las construcciones posesivas. Como por ejemplo, la lengua cahuilla (lengua uto-azteca) que presenta dos tipos de clasificadores. Por un lado, un conjunto de clasificadores llamados 'temporales' que son similares a los clasificadores relacionales, en tanto señalan la forma en que un ítem puede ser manejado. Así, el nombre meñikiš 'poroto' recibe un clasificador diferente según sea arrancado del árbol o recogido del suelo. Por otro lado, un conjunto de clasificadores que se refiere a las propiedades inherentes de un nombre poseído (AIKHENVALD, 2003, p. 143-144). 


\subsection{La posesión}

De acuerdo con Heine (1997) ciertos dominios de la gramática, como por ejemplo el dominio de la posesión, sólo pueden ser explicados satisfactoriamente si se consideran los esquemas o estructuras semántico-cognitivas de las que derivan. Estos esquemas se llaman esquemas evento y presentan las siguientes características:

- Tienen forma proposicional.

- Están relacionados a dominios ontológicos como acción, ubicación, etc.

- Son abstraídos a partir de las formas en que experimentamos nuestro entorno y describimos nuestras experiencias al comunicarnos con los demás.

Heine sostiene que la posesión es un dominio de conceptualización humana relativamente abstracto, que requiere ser derivado de dominios más concretos. Dichos dominios tienen que ver con experiencias básicas, relacionadas con lo que se hace (acción), dónde se está (ubicación), con quien se está (compañía), qué existe (existencia). Estas experiencias se denominan esquemas evento.

La mayoría de las construcciones posesivas pueden derivarse históricamente de ocho esquemas: esquema acción, esquema ubicación, esquema compañía, esquema genitivo, esquema meta, esquema tópico, esquema fuente y esquema ecuación. Para ilustrar esto consideremos el siguiente ejemplo propuesto por el autor: "Un auto que me pertenece, puede ser descripto como algo que regularmente está cerca de mí (ubicación); algo que yo manejo (acción); algo que me acompaña (compañía); o que está para mí (existencia)” (HEINE, 1997, p. 76).

En el cuadro 2 presentamos las características de cada uno de estos esquemas y las construcciones posesivas ${ }^{9}$ que pueden derivarse de ellos. 


\section{CUADRO 2}

Descripción de los esquemas eventos y ejemplo de las construcciones posesivas derivadas

\begin{tabular}{|c|c|c|c|}
\hline $\begin{array}{c}\text { Esquemas } \\
\text { (Descripción en } \\
\text { fórmula X: poseedor } \\
\text { Y: poseído) }\end{array}$ & Estructura proposicional & $\begin{array}{c}\text { Construcciones } \\
\text { posesivas } \\
\text { derivadas }\end{array}$ & Ejemplo $^{10}$ \\
\hline $\begin{array}{l}\text { Esquema } \\
\text { acción } \\
X \text { takes } Y>X \\
\text { has, own } Y\end{array}$ & $\begin{array}{l}\text { Según este esquema la noción de } \\
\text { posesión predicativa es derivada } \\
\text { de un estructura proposicional } \\
\text { que supone un agente, un } \\
\text { paciente y alguna acción o } \\
\text { actividad. X es codificado como } \\
\text { sujeto y el poseído como objeto/ } \\
\text { complemento. Los verbos pue- } \\
\text { den ser activos (agarrar, tomar, } \\
\text { apropiarse de, etc.) o inactivos } \\
\text { y/o no dinámicos (sostener, } \\
\text { adquirir, obtener, etc.). }\end{array}$ & $\begin{array}{l}\text { Construcciones } \\
\text { con el verbo tener } \\
\text { y construcciones } \\
\text { de pertenencia. } \\
\text { (Este esquema no } \\
\text { puede dar origen a } \\
\text { construcciones de } \\
\text { posesión atributiva) }\end{array}$ & $\begin{array}{l}\text { 1. Portugués } \\
\text { O menino tem fome } \\
\text { the child takes/has hunger } \\
\text { 'The child is hungry' } \\
\text { 2. Nama } \\
\text { Kxoe p ke 'auto sa 'uu } \\
\text { Person M TOP car F take } \\
\text { haa } \\
\text { PERF } \\
\text { 'The man has the car' } \\
\text { (Lit: The man has taken } \\
\text { a/the car) } \\
\text { 3. Dullay } \\
\text { Ló'ó an-sheeg-a } \\
\text { cow I-carry-1.SG.IPEV } \\
\text { 'I have a cow' }\end{array}$ \\
\hline $\begin{array}{l}\text { Esquema } \\
\text { ubicación } \\
\text { Y is at X's place> } \\
\mathrm{X} \text { has, own Y }\end{array}$ & $\begin{array}{l}\text { Las construcciones posesivas que } \\
\text { derivan de este esquema presentan } \\
\text { la siguiente estructura sintáctica: } \\
\text { Y es codificado como sujeto y X } \\
\text { como complemento locativo. } \\
\text { El predicado es una cópula loca- } \\
\text { tiva o un verbo estativo (estar en, } \\
\text { quedarse, sentarse, etc.). El } \\
\text { predicado no tiene que ser } \\
\text { obligatoriamente un ítem verbal } \\
\text { (véase Ej. 5). } \\
\text { Este esquema tiene dos sub- } \\
\text { esquemas que pueden describirse } \\
\text { con las siguientes fórmulas: } \\
\text { a. Y is at X's home (véase Ej. 6) } \\
\text { b. Y is at X's body-part } \\
\text { (véase Ej. 7). }\end{array}$ & $\begin{array}{l}\text { Construcciones con } \\
\text { el verbo tener y } \\
\text { posesión atributiva } \\
\text { (véase Ej. } 5 \text { y } 8 \text { ). } \\
\text { Nota: este esquema } \\
\text { es uno de los más } \\
\text { usados para expresar } \\
\text { posesión atributiva: } \\
\text { X es conceptualizado } \\
\text { como el lugar donde } \\
\text { Y está ubicado. }\end{array}$ & $\begin{array}{l}\text { 4. Estonio } \\
\text { Isal on } \\
\text { father.ADESSIVE 3.SG.be } \\
\text { raamat } \\
\text { book.NOM } \\
\text { 'Father has (a) book' (Lit: } \\
\text { 'The book is at father') } \\
\text { 5. Kxoé } \\
\text { Ti ò /oán-djì } \\
\text { I at child-F.PL } \\
\text { 'My doughters' (Lit: } \\
\text { female chlidren at } \\
\text { my place) } \\
\text { 6. S o } \\
\text { Mek Auca eo-a kus -in } \\
\text { NEG.be:at Auca home-LOC } \\
\text { 'Auca has no clothes' (Lit: } \\
\text { Skins are not at Auca's } \\
\text { house) }\end{array}$ \\
\hline
\end{tabular}




\begin{tabular}{|c|c|c|c|}
\hline $\begin{array}{l}\text { Esquema } \\
\text { compañía } \\
\mathrm{X} \text { is with } \mathrm{Y}> \\
\mathrm{X} \text { has, own } \mathrm{Y}\end{array}$ & $\begin{array}{l}\text { De acuerdo con este esquema X } \\
\text { se codifica como sujeto e Y como } \\
\text { complemento comitativo y es } \\
\text { conceptualizado como una clase } \\
\text { de compañero. }\end{array}$ & $\begin{array}{l}\text { Construcciones con } \\
\text { el verbo tener y } \\
\text { posesión atributiva. } \\
\text { Nota: este esquema } \\
\text { es en gran medida } \\
\text { usado para cons- } \\
\text { trucciones con el } \\
\text { verbo tener pero } \\
\text { existen algunas } \\
\text { instancias de } \\
\text { posesión atributiva } \\
\text { (véase Ej. 12). }\end{array}$ & 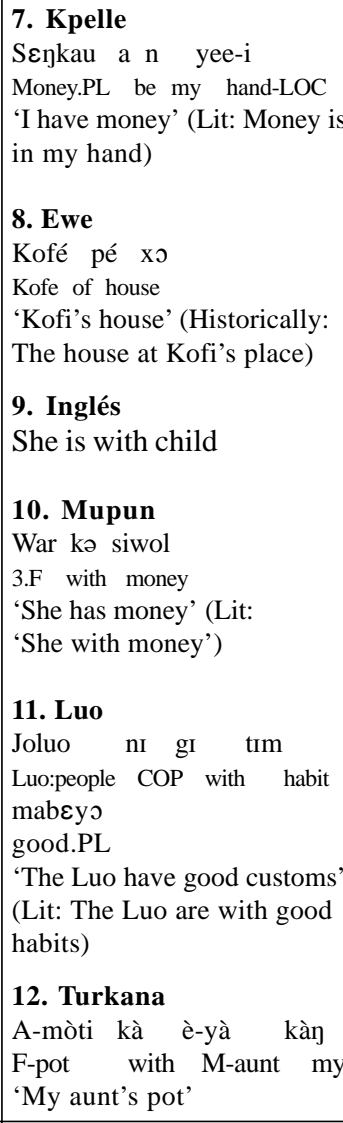 \\
\hline $\begin{array}{l}\text { Esquema } \\
\text { genitivo } \\
\text { X's Y exists > } \\
\text { X has, own Y }\end{array}$ & $\begin{array}{l}\text { Este esquema se caracteriza por } \\
\text { el hecho de que X es codificado } \\
\text { como un modificador genitivo de } \\
\text { Y; e Y es codificado como sujeto. }\end{array}$ & $\begin{array}{l}\text { Construcciones con } \\
\text { el verbo tener. }\end{array}$ & $\begin{array}{l}\text { 13. Turco } \\
\text { Kitab-im var } \\
\text { book-my existance } \\
\text { 'I have a book' (Lit: my } \\
\text { book exits) } \\
\text { 14. Anywa } \\
\text { Dá cí--`` } \\
\text { exist wife:of-3: SG } \\
\text { 'He has a wife' (Lit: His wife } \\
\text { exists) }\end{array}$ \\
\hline
\end{tabular}




\begin{tabular}{|c|c|c|c|}
\hline $\begin{array}{l}\text { Esquema meta } \\
\mathrm{Y} \text { exits to/for } \mathrm{X}> \\
\mathrm{X} \text { has, own } \mathrm{Y}\end{array}$ & $\begin{array}{l}\text { Este esquema presenta típica- } \\
\text { mente, un verbo de existencia } \\
\text { o de posición. X es codificado } \\
\text { como dativo/benefactivo o } \\
\text { como expresión de caso } \\
\text { objetivo, e Y como sujeto. }\end{array}$ & $\begin{array}{l}\text { Construcciones con } \\
\text { el verbo tener, } \\
\text { construcciones de } \\
\text { pertenencia. (Véase } \\
\text { Ej.16) y posesión } \\
\text { atributiva (véase } \\
\text { Ej.17 y 18). } \\
\text { Nota: este es el } \\
\text { único esquema que } \\
\text { permite la formación } \\
\text { de los tres tipos de } \\
\text { construcciones } \\
\text { posesivas. }\end{array}$ & $\begin{array}{l}\text { 15. Quechua (boliviano) } \\
\text { Waska tiya -puwan } \\
\text { rope exist-for me } \\
\text { 'I have a rope' } \\
\text { 16. Francés } \\
\text { Le livre est à moi } \\
\text { the book is to me } \\
\text { 'The book belongs to me' } \\
\text { 17. Aranda } \\
\text { Toby-ke alere } \\
\text { Toby-DAT child } \\
\text { 'Toby's child' } \\
\text { 18. Inglés pidgin del oeste } \\
\text { de África } \\
\text { Wok-tíng fo mésan } \\
\text { 'Tools of/for the mason' }\end{array}$ \\
\hline $\begin{array}{l}\text { Esquema } \\
\text { tópico } \\
\text { As for X, Y } \\
\text { (of X) exists > } \\
\text { X has, own Y }\end{array}$ & $\begin{array}{l}\text { Presenta a X como tema o tó- } \\
\text { pico -en posición inicial- pero } \\
\text { a la vez figura como un modi- } \\
\text { ficador posesivo de Y. }\end{array}$ & $\begin{array}{l}\text { Construcciones con } \\
\text { el verbo tener y } \\
\text { posesión atributiva } \\
\text { (véase Ej.19 y 20). } \\
\text { Nota: este esquema } \\
\text { es uno de los más } \\
\text { comunes para la } \\
\text { expresión de } \\
\text { construcciones } \\
\text { atributivas. }\end{array}$ & $\begin{array}{l}\text { 19. Lango } \\
\text { Òkélò gwók' kere pé } \\
\text { Okelo dog-his 3.NEG.exist } \\
\text { 'Okelo doesn't have a dog' } \\
\text { 20. Kairiru } \\
\text { Nur yaqal qajuo-ny } \\
\text { Nur he cousin-his } \\
\text { 'Nur's cousin' } \\
\text { 21. Afrikáans } \\
\text { Die boer se huis } \\
\text { the farmer his house } \\
\text { 'The farmer's house' }\end{array}$ \\
\hline $\begin{array}{l}\text { Esquema } \\
\text { fuente } \\
\text { Y exists from } \mathrm{X}> \\
\mathrm{X} \text { has, own } \mathrm{Y}\end{array}$ & $\begin{array}{l}\text { En este esquema X es expresado } \\
\text { como un participante ablativo, } \\
\text { codificado generalmente como } \\
\text { un marcador que denota nociones } \\
\text { como 'from', 'off' y 'out of. }\end{array}$ & $\begin{array}{l}\text { Posesión } \\
\text { atributiva. }\end{array}$ & $\begin{array}{l}\text { Ejemplos comunes con este } \\
\text { tipo de esquema incluyen } \\
\text { expresiones con el uso del } \\
\text { genitivo of del inglés, el } \\
\text { genitivo von del alemán y el } \\
\text { genitivo de de las lenguas } \\
\text { romances. }\end{array}$ \\
\hline $\begin{array}{l}\text { Esquema } \\
\text { ecuación } \\
\text { Y is X's } \\
\text { (property) > } \\
\text { Y belongs to X }\end{array}$ & $\begin{array}{l}\text { En este esquema } \mathrm{X} \text { es presentado } \\
\text { como un modificador genitivo. } \\
\mathrm{Y} \text { es codificado como sujeto. }\end{array}$ & $\begin{array}{l}\text { Construcciones de } \\
\text { pertenencia. }\end{array}$ & $\begin{array}{l}\text { 22. Swahili } \\
\text { Gari ni yangu } \\
\text { car is mine } \\
\text { 'The car is mine' } \\
\text { 23. Inglés } \\
\text { The book is Jhon's }\end{array}$ \\
\hline
\end{tabular}


Algunas notas respecto a los esquemas:

- El esquema acción es el único que presenta un núcleo predicativo con contenido lexical. Los otros esquemas tienen núcleos predicativos simplificados, en tanto expresan contenidos como ubicación, existencia, ecuación o identificación y compañía.

- Los esquemas acción y ubicación son estructuralmente básicos mientras que los otros esquemas son extendidos, porque tienen un participante adicional: el esquema compañía tiene un adjunto comitativo, el esquema meta tiene un adjunto dativo, alativo o benefactivo y tanto el esquema genitivo como el esquema ecuación, tienen un modificador genitivo.

- Los esquemas acción y compañía codifican al poseedor como sujeto de la cláusula mientras que los otros esquemas codifican al poseído como sujeto y al poseedor como caso oblicuo: locativo, dativo, o modificador genitivo.

- Es posible hacer algunas generalizaciones respecto a las nociones de posesión ${ }^{11}$ que un esquema puede expresar: el esquema ubicación está asociado generalmente a expresar posesión física o temporal; por su parte, los esquemas objetivo, genitivo y tópico están asociados con expresiones de posesión permanente e inalienable y el esquema compañía, está asociado a expresiones de posesión física o temporal y generalmente, con posesión alienable.

\section{Antecedentes}

Las referencias que poseemos sobre los clasificadores de posesión en wichí son las gramáticas de Viñas Urquiza (1974) y Terraza (2009) y un artículo de Golluscio (1993). Como veremos, sólo las formas ka y lo han sido estudiadas y analizadas en estos trabajos.

Según Viñas Urquiza (1974) kä y lä son pronombres posesivos para indicar la posesión de personas y objetos, y la posesión de animales respectivamente. Según la autora estos pronombres pueden combinarse con los prefijos de persona y con el sufijo de plural.

Por su parte, Golluscio (1993) sostiene que ka es un marcador de aptitud para ser poseído y que la es un clasificador exclusivo para nombres referidos a animales.

Finalmente, Terraza (2009) es quien propone tratar a $k a$ y lo como clasificadores de posesión que aparecen en construcciones de posesión con 
nombres alienables. Esta autora señala que lo es un clasificador específico que se usa para categorizar nombres referidos a animales mientras que $k a$ es un clasificador general. Ambos clasificadores son analizados como palabras independientes por los siguientes motivos: i. los clasificadores, al igual que los nombres, pueden co-ocurrir con prefijos posesivos y las marcas de plural; ii. entre el clasificador y el nombre pueden estar presentes otros elementos y iii. fonológicamente son portadores del acento.

Respecto a ko Terraza menciona que es una forma especial usada en los mismos contextos que los clasificadores pero con el significado aproximado de la pertenencia de.

\section{Análisis}

En esta primera instancia de la investigación detallamos las características morfosintácticas de los clasificadores y brindamos una primera aproximación sobre las diferencias semánticas en el uso de los mismos.

El análisis está organizado en tres partes. En 3.1 mencionamos brevemente cómo se manifiesta en wichí la posesión alienable/inalienable. En 3.2 describimos las particularidades de los clasificadores y presentamos sus compatibilidades. En 3.3, analizamos el comportamiento de los clasificadores y su uso. Finalmente, en 3.4 presentamos las diferencias semánticas entre las construcción posesivas con ka, lo y ko.

\subsection{Posesión alienable e inalienable en wichí}

El wichí presenta distinción gramatical entre posesión alienable e inalienable. La posesión inalienable se manifiesta mediante la presencia de un poseedor obligatorio, ya sea, mediante la presencia de un prefijo posesivo en el nombre poseído o mediante un nombre independiente (ejemplo (1)). En (1b) podemos ver que la presencia del prefijo posesivo es obligatoria aunque esté presente el nombre poseedor. La posesión alienable se indica mediante la presencia de un clasificador que antecede al nombre poseído: el clasificador $k a$ para todos los nombres alienables en general y el clasificador lo para nombres referidos a animales domésticos. En estos casos el clasificador es determinado por los prefijos de posesión (ejemplo (2a) y (3a)). 
(1) (a) $\mathbf{u}$-let $\int \mathrm{e}-\mathrm{j} \quad$ ojta-hes

1POS/sG-pierna-PL ¿dolor?-PL

'Me duelen las piernas' (Lit: mis piernas dolorosas)

(b) tam la-wet

3PRO/sG 3POS-lugar/casa

'La casa de él' (Lit: él su casa)

\subsection{Particularidades y compatibilidades de los clasificadores posesión}

En nuestro corpus hemos identificado tres clasificadores de posesión $k a$, lo y ko que podemos considerar como pertenecientes a una misma clase, puesto que como vemos en (2) a (4) se excluyen mutuamente.

En (2) la posesión del nombre pem ‘bombo' puede ser indicada por ka y ko pero no por ambos clasificadores en la misma oración.

(2) (a) toxa roli la-ka pem PROdem Roli 3POS-CL bombo

'Este es el bombo de Roli' (Lit: esto Roli su bombo)

(b) ułam ni-tiłame hop pem to roli (la)-ko

1PRO/sG 1sG-conservar FOC bombo SUB Roli 3POS-CL

'Yo tengo el bombo de Roli'

(Lit: yo conservo bombo que (es) pertenencia de Roli)

(c) *ułam ni-tiłame hop la-ka pem to roli (la)-ko

En (3) el nombre poseído sinox 'perro' puede ser categorizado por $k a$ o por lo pero no por ambos en la misma oración.

(3) (a) u-lo $\operatorname{sinox}$

1POS/sG-CL perro

'Mi perro' (Lit: mi mascota perro)

(b) u-ka sinox

1POS/sG-CL perro

'Mi perro'

(c) *u-lo u-ka sinox

Finalmente en (4), vemos que tanto lo como ko pueden presentar la misma distribución y ubicarse después del nombre poseedor pero no pueden categorizar 
la misma clase de nombres: $k o$ no puede categorizar nombres referidos a animales y lo no puede categorizar nombres referidos a objetos.

(4) (a) sinox $\emptyset$-jajonli huu serxjo la-lo perro 3-perseguir gallina Sergio 3POS-CL 'El perro persigue a las gallinas de Sergio' (Lit: perro persigue (a la) gallina mascota (de) Sergio)

(b) notsas $\emptyset$-t' $\mathrm{ek}^{\mathrm{w}} \mathrm{e}$ lax ${ }^{\mathrm{w}} \mathrm{ul}$ maestalu la-ko niños 3-buscar flauta maestra 3POS-CL 'Los niños están buscando la flauta de la maestra' (Lit: niños buscan flauta pertenencia de (la) maestra)

(c) *sinox $\emptyset$-jajonti huu sergjo la-ko

$*$ notsas $\emptyset-\mathrm{t}^{\mathrm{2}} \mathrm{ek}^{\mathrm{w}} \mathrm{e}$ lax ${ }^{\mathrm{w}} \mathrm{ul}$ maestalu la-lo

En principio, podemos analizar a ka, ko y lo como clasificadores de posesión nominales, puesto que aparecen en construcciones posesivas categorizando a un nombre. No obstante, el análisis de sus compatibilidades nos permite considerar la posibilidad de establecer tres subclases diferentes.

\subsubsection{El clasificador $k a$}

El clasificador $k a$ modifica a nombres alienables y siempre aparece antes del nombre poseído. En (5) ka puede ir acompañado de los prefijos de posesión pero no tiene marca de plural (ejemplo (5b)).

(5) (a) ułam uj-chen? u-ka jote

1PRO/sg 1sG-cargar 1POS/sG-CL botija

'Yo cargo mi botija -con agua-'

(b) u-ku la-ka $\operatorname{sino}(\mathrm{x})-\mathrm{s}$

1POS/sG-mamá 3sG-CL perro-PL

'Los perros de mi mamá' (Lit: mi mamá su perros)

\subsubsection{El clasificador lo}

El clasificador lo tiene el significado de mascota y es usado únicamente con animales domésticos como sinox 'perro', mitsi ‘gato', ele 'loro', tsunatax 'oveja', yelatax ‘caballo' huu 'gallina'. Por lo tanto, animales como mawu 'zorro', 
wahat 'pez', chilaji 'paloma de la ciudad' y ałetax 'yacaré' no pueden ser determinados por $l o$.

Generalmente es prenominal pero también puede ser postnominal (véase ejemplo (4a)).

(6) (a) tam i-t 0 la-lo tulu 3PRO/sg 3-llevar POS-CL vaca 'Éllleva su (mascota) vaca'

(b) $\mathrm{u}-\mathrm{lo}-\mathrm{j} \quad$ sino-s 1POS/sG-CL-PL perro-PL 'Mis (mascotas) perros'

Como vemos en (6) lo puede ir acompañado de prefijos de posesión y del sufijo de plural. También hemos registrado ejemplos en los que lo puede combinarse con el afijo diminutivo $-\chi^{\mathrm{w}} a$. En (7) la presencia del diminutivo en $l o$ o en el nombre poseído no produce cambio de significado.
(7)
(a) $\mathrm{u}-\mathbf{l o}-\mathbf{x}^{\mathrm{w}} \mathbf{a}$
mitsi
1POS/sG-CL-DIM gato
(b) u-lo
1POS/sG-CL gato-DIM
'Mi gatito' (Lit: mi mascotita gato)
'Mi (mascota) gatito'

\subsubsection{El clasificador $k o$}

El clasificador ko tiene el significado de la pertenencia de y es generalmente postnominal, ${ }^{12}$ puede aparecer después del nombre poseedor (en la mayoría de los ejemplos) o después del nombre poseído.

El nombre poseído puede ser alienable, como hunat 'tierra' y jawes 'llaves' o inalienable como kos 'fruta' y wet 'lugar'. En (8) ko puede combinarse con los prefijos de persona y no tiene marca de plural (ejemplo (8d)).

(8) (a) hap suwele $\emptyset$-ihi hunat witfi (la)-ko FOC criollo 3-estar tierra wichí/gente 3POS-CL

'Los criollos están en la tierra (pertenencia) de los wichís'

(b) portero to i-tiłame jawes [to eskwela la-ko] portero SUB 3-conservar llaves SUB escuela 3POS-CL 'El portero conserva las llaves de la escuela' (Lit: portero que conserva (las) llaves [que (son) pertenencia de la escuela] 
(c) ułam uj-hona serxjo la-kos [to la-ko]

1PRO/sg 1sG-usar Sergio 3POS-fruta SUB 3POS-CL

'Yo uso la fruta que pertenece a Sergio -que él sembró-' (Lit: yo uso Sergio su fruta [que (es) pertenencia de él]

(d) ułam u-sikaje witji la-wet-es la-ko 1PRO/sg 1sG-visitar wichí/gente 3POS-lugar-PL 3POS-CL 'Yo visité el lugar (pertenencia) de los wichí'

Podemos resumir lo señalado hasta aquí en el cuadro 3. Como podemos ver, $k o$ se distingue de $k a$ y lo por su distribución (ya que generalmente es postnominal) y por el hecho de que no categoriza al nombre poseído. Por el momento, podemos pensar que ko categoriza más bien la relación de posesión entre el poseedor y poseído. Cabe aclarar que los ejemplos registrados con $k o$ son pocos en comparación con los ejemplos de ka y lo.

\section{CUADRO3}

Características de los clasificadores de posesión ka, lo y ko

\begin{tabular}{|c|c|c|c|c|}
\hline \multicolumn{2}{|c|}{ Características } & $k a$ & ko & lo \\
\hline \multirow{4}{*}{ Compatibilidades } & $\begin{array}{l}\text { Combinación con } \\
\text { prefijos de posesión }\end{array}$ & Sí & Sí & Sí \\
\hline & $\begin{array}{l}\text { Combinación con } \\
\text { sufijo de plural }\end{array}$ & No & No & Sí \\
\hline & $\begin{array}{l}\text { Combinación con } \\
\text { diminutivo }\end{array}$ & No & No & Sí \\
\hline & Categorización & $\begin{array}{l}\text { Categoriza al } \\
\text { nombre poseído } \\
\text { (nombre } \\
\text { alienable). }\end{array}$ & $\begin{array}{l}\text { Aparentemente, } \\
\text { categoriza la } \\
\text { relación de } \\
\text { posesión entre } \\
\text { el nombre } \\
\text { poseído y el } \\
\text { nombre poseedor. }\end{array}$ & $\begin{array}{l}\text { Categoriza al } \\
\text { nombre poseído } \\
\text { (referido siempre } \\
\text { a un animal } \\
\text { doméstico). }\end{array}$ \\
\hline \multicolumn{2}{|c|}{ Distribución } & Prenominal & $\begin{array}{l}\text { Postnominal } \\
\text { (aparece después } \\
\text { del nombre } \\
\text { poseedor o } \\
\text { después del } \\
\text { nombre poseído). }\end{array}$ & $\begin{array}{l}\text { Prenominal } \\
\text { (generalmente) y } \\
\text { postnominal. }\end{array}$ \\
\hline
\end{tabular}




\subsection{Análisis del comportamiento y uso de ka, ko y lo}

\subsubsection{Los clasificadores ka y lo}

En base a los datos presentados en 3.2 podemos plantear como primera hipótesis que $\mathrm{ka}$ y lo categorizan a un nombre poseído alienable. $\mathrm{Ka}$ es un clasificador general que categoriza a todos los nombres alienables independientemente de sus características y de la forma en que éstos pueden ser poseídos. Y lo es un clasificador específico que categoriza a nombres referidos únicamente a animales domésticos. Así en (9) podemos ver que un mismo nombre alienable huu 'gallina' puede ser categorizado por $k a$ (en tanto clasificador general) y por lo (en tanto clasificador específico) sin producir cambios semánticos.
(9) (a) u-ka huu 1sG-CL gallina 'Mis gallinas'
(b) u-lo-j huu 1sG-CL-PL gallina 'Mis (mascotas) gallinas'

No obstante, en (10) podemos ver que el nombre tulu 'vaca' adquiere el significado de 'carne' si es usado con $k a$.
(10) (a) u-lo tulu 1sG-CL vaca 'Mi vaca'
(b) u-ka tulu 1sG-CL vaca 'Mi carne -que compré-

También, hemos observado que el uso de $k a$ con nombres referidos a elementos de la naturaleza como tierra, monte y agua cambia el significado léxico para indicar la forma en que estos elementos pueden ser poseídos o adquiridos por las personas. Como vemos en el cuadro 4 hunat 'tierra' y taji 'monte' son elementos que las personas pueden adquirir y poseer en tanto habiten o trabajen en ellos; así mismo, el agua puede ser propiedad de las personas si es recogida. ${ }^{13}$

\section{CUADRO 4}

Significado de los nombres hunat, taji e inot con y sin ka

\begin{tabular}{|l|l|}
\hline Sin clasificador $k a$ & Con clasificador $k a$ \\
\hline hunat 'tierra' & hunat 'jardín, parcela' -lugar que pertenece a la persona- \\
\hline taji 'monte' & taji 'lugar donde siembro' \\
\hline inot 'agua' & inot 'agua recogida' \\
\hline
\end{tabular}


Incluso, el uso de $k a$ con nombres referidos a personas como hinu 'hombre' y atsina 'mujer' indica relaciones de parentesco: $k a$ hinu 'esposo, enamorado / hijo varón' y ka tsina 'esposa, mujer, amante / hija mujer'. ${ }^{14}$

Estos datos respecto al uso de $k a$ nos permiten plantear como segunda hipótesis que $k a$ no sólo categoriza de manera general a nombres poseídos alienables, sino también el tipo de relación que se establece entre el poseedor y el poseído: ka tsinaj ‘mi mujer' (relación de parentesco) ka tulu 'mi carne’ (relación de propiedad adquirida) $k a$ hunat ‘mi parcela’ (relación de propiedad adquirida).

\subsubsection{El clasificador ko: diferencias y similitudes con ka y lo}

Respecto a las compatibilidades de estos clasificadores podemos señalar que $k o$ y lo, a diferencia de $k a$, pueden combinarse con los prefijos de posesión constituyendo sintagmas y ser usados como referencias anafóricas.

a) Posesivo prefijado + $k o=$ 'pertenencia tuya, de él, de ellos...'

b) Posesivo prefijado + lo + suf. PL + suf. DIM = 'mi, tu, de él. . mascota(s) / mascotita'

Además, tanto ko como lo pueden combinarse con los morfemas jen 'hacer' y - $a$ 'verbalizador' y ser usados con el significado de adueñarse y adoptar -hacer de un animal una mascota- respectivamente. ${ }^{15}$ No hemos registrado ejemplos en los que $k a$ se combine con esos morfemas.

(11) jen-ko-ja mesa hacer-CL-VERB mesa

'(él) se adueña de la mesa'

(12) jen-lo-ya mitsi hacer-CL-VERB gato

'(él) adopta el gato -hacer del gato su mascota-'

Respecto a los contextos de uso, podemos señalar que $k o$ y lo no pueden ser usados en los mismos contextos, puesto que como vimos en (4) ko no es usado con nombres referidos a animales; mientras que $k o$ y $k a$ sí pueden ser usados en los mismos contextos; es decir, aparecer en construcciones posesivas con los mismos nombres. No obstante, hay algunas diferencias respecto al uso de $k a$ y $k o$ : 
i. Aunque se usan en los mismos contextos el orden de los mismos en la construcción es diferente: $k a$ siempre precede al nombre poseído mientras que $k o$ es generalmente postnominal: sigue al nombre poseedor o al nombre poseído.

ii. ka y ko no siempre pueden ser usados en los mismos contextos. En (13) podemos ver que el nombre alienable hanox ${ }^{w} a$ 'bebé' es categorizado por $k a$ pero no por $k o$.

(13) tamil la-ka (ha)noxwa

3PRO/PL POS-CL bebé

'Bebé de ellos' (Lit.: ellos su bebé)

* hanoxwa (to) łamil la-ko

En el cuadro 5 presentamos a modo de comparación el uso de construcciones posesivas con ka y ko (la $\mathrm{x}$ indica que el nombre poseído aparece en la construcción). En las construcciones posesivas con ko el nombre poseído (referido generalmente a objetos o materiales) puede ser alienable o inalienable.

\section{CUADRO5}

Comparación entre el uso de $k a$ y ko

\begin{tabular}{|c|c|c|c|}
\hline Nombre poseído & $\begin{array}{c}\text { En construcciones } \\
\text { con ka y ko }\end{array}$ & $\begin{array}{l}\text { En construcciones } \\
\text { sólo con } k a\end{array}$ & $\begin{array}{c}\text { En construcciones } \\
\text { sólo con ko }\end{array}$ \\
\hline pem ‘bombo’ (alienable) & $\mathrm{x}$ & & \\
\hline helek ‘yica’ (alienable) & $\mathrm{x}$ & & \\
\hline jawes ‘llaves’ (alienable) & $\mathrm{x}$ & & \\
\hline tsinis ‘peine’ (alienable) & $\mathrm{x}$ & & \\
\hline hanoxwa 'bebé' (alienable) & & $\mathrm{x}$ & \\
\hline mitsi ‘gato’ (alienable) & & $\mathrm{x}$ & \\
\hline atsina 'mujer' (alienable) & & $\mathrm{x}$ & \\
\hline inot ‘agua'(alienable) & & $\mathrm{x}$ & \\
\hline nisox ‘zapato’ (inalienable) & & & $\mathrm{x}$ \\
\hline kos 'frutas' (inalienable) & & & $\mathrm{x}$ \\
\hline wet 'lugar' (inalienable) & & & $\mathrm{x}$ \\
\hline
\end{tabular}


Todo lo mencionado hasta aquí nos lleva a plantear la siguiente hipótesis: los clasificadores integran, de acuerdo con Aikhenvald, un mismo sistema que indica tanto las características del ítem poseído como la relación que se establece entre el poseedor y el poseído.

\section{CUADRO 6}

Integración de los clasificadores de posesión y relacionales en un sistema

\begin{tabular}{|l|l|}
\hline Clasificadores & \multicolumn{1}{c|}{ Semántica } \\
\hline$K a$ & General (para todos los nombres alienables) \\
\hline$L o$ & Animales -que pueden ser domesticados- \\
\hline$K o$ & $\begin{array}{l}\text { Objetos y elementos -que tienen un determinada función y por ello } \\
\text { pueden ser manipulados o transportados (como bombo, mochila, yica, } \\
\text { etc.), transformados o usados para ciertos fines por el poseedor (como } \\
\text { tierra y lugar). }\end{array}$ \\
\hline
\end{tabular}

Pero si ka, ko y lo integran un mismo sistema ¿Por qué ko es usado independientemente de la distinción gramatical alienable/inalienable, mientras que $k a$ y lo son usados sólo con nombres alienables? Si $k a$ y ko pueden aparecer en los mismos contextos ¿qué diferencia existe en el uso de uno y otro?

\subsection{Semántica de las construcciones posesivas con ka, ko y lo}

\subsubsection{Nociones de posesión en construcciones con ka y lo}

De acuerdo con Heine, podemos pensar que las construcciones posesivas con los clasificadores $k a$ y lo derivan del esquema tópico: para X, Y (de X) existe $>\mathrm{X}$ tiene $\mathrm{Y}$. En los ejemplos que mostramos con $k a$ y lo el poseedor se presenta como tópico (aparece siempre en posición inicial) y a la vez, es modificador genitivo del poseído. El poseído es el núcleo de la frase nominal.

En base a este esquema podemos decir que en (14) el poseído existe o está porque el poseedor lo usa para ciertos fines -ejemplos (14a) y (14d)-, está asociado al ítem poseído -ejemplo (14e)-, interacciona con el poseído -ejemplo (14b)- o mantiene una relación de parentesco con el mismo -ejemplo (14c)-. 
(14) X prefijo-ka Y

(a) $\mathrm{X}=[+$ humano $] \mathrm{Y}=[$-humano -animado $]$

toxa serxjo la-ka tsinis

PROdem Sergio 3POS-CL peine

'Este es el peine de Sergio' (Lit: este Sergio su peine)

$\emptyset$-jenti la-ka wuna

3sG-hacer 3POS/sG-CL sombrero

'Fabrica su sombrero -con papel-'

(b) $\mathrm{X}=[+$ humano $] \mathrm{Y}=[$ +animado: animal $]$

u-ku la-ka $\operatorname{sino}(\mathrm{x})-\mathrm{s}$

1POS/sG-mamá 3sg-CL perro-PL

'Los perros de mi mamá' (Lit: mi mamá su perros)

(c) $\mathrm{X}=[+$ humano $] \mathrm{Y}=[+$ humano $]$

ułam u-ije serxjo la-ka wichí

PRO/sg 1sG-estar Sergio 3POS-CL persona/gente

'Yo estoy con la familia de Sergio' (Lit.: yo estoy Sergio su gente)

(d) $\mathrm{X}=[$ +animado: animal $\mathrm{Y}=[$-animado $]$

u-ka mitsi la-ka pelutax

1POS/sG -CL gato 1POS/sG-CL pelota

'Mi gato tiene su pelota' (Lit.: mi gato su pelota)

(e) $\mathrm{X}=[$-animado $\mathrm{Y}=[$-animado $]$

eskwela la-ka liblu

escuela 3POS-CL libro

'Libro de la escuela' (Lit.: escuela su libro)

Por su parte, en (15) el poseído existe o está porque el poseedor lo domesticó.

(15) $\mathrm{X}$ prefijo-lo $\mathrm{Y}$

(a) $\mathrm{X}=[+$ humano $] \mathrm{Y}=[$ +animado: siempre animal $]$

fam i-t jo la-lo tulu

3PRO/sg 3-llevar 3POS-CL vaca

'Él lleva su (mascota) vaca' 


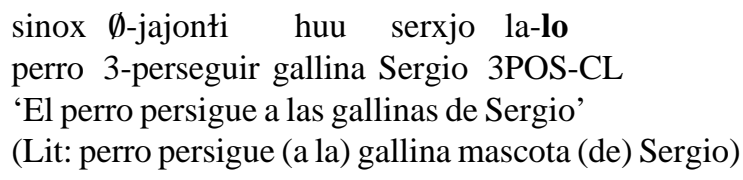

Los clasificadores $k a$ y lo son usados en construcciones posesivas atributivas que pueden expresar nociones de posesión permanente como en (15) y (16) o temporales y físicas como en (17).

(16) (a) toxa serxjo la-ka tsinis PROdem Sergio 3POS-CL peine 'Este es el peine de Sergio' (Lit: este Sergio su peine)

(b) i-połi mak la-t $\int i l a \quad$ la-ka pem 3-tocar cosa 3POS-hermano 3POS-CL bombo '(Él) toca el bombo de su hermano' (Lit: toca cosa (que es) de su hermano su bombo)

(c) $\mathrm{u}-\left(\mathrm{t}^{\mathrm{i}} \mathrm{i}\right) \mathrm{t} \int \mathrm{ujeti}$ to ten(e)k-haj iglesja to ka-majek 1POS/sG-cantar SUB canción-PL iglesia SUB CL-cosa '(Yo) canto canciones de la iglesia' (Lit.: yo canto canciones (que son) cosa (pertenencias) de la iglesia.

(17) (a) i-tatsi la-ka papel $\emptyset$-jopil 3-mandar 3POS-CL papel 3-ir '(Ella) manda una carta' (Lit: manda su papel se va)

(b) u-ka tulu 1sG-CL vaca 'Mi carne -que compré-

De acuerdo con Heine (véase nota 11) la noción de posesión permanente indica que el poseído es propiedad del poseedor. Pero en el caso de las construcciones con $k a$ que expresan posesión permanente, el ítem poseído puede o no pertenecer al poseedor. De acuerdo con la información brindada por nuestro informante wichí, es posible decir tam la-ka mati 'el mate de él/ella' (independientemente de que el mate pertenezca o no al poseedor) porque 'veo' que el poseedor usa en el momento el mate o porque 'veo' que el poseedor siempre está con el mate y lo usa ${ }^{16}$ (independientemente de que lo haya comprado, fabricado o se lo hayan regalado o prestado). 


\subsubsection{Nociones de posesión en construcciones con ko}

Las construcciones posesivas con $k o$, a diferencia de $k a$ y lo, derivan del esquema ecuación: $\mathrm{Y}$ es (propiedad) de $\mathrm{X}>\mathrm{Y}$ pertenece a $\mathrm{X}$. Al igual que en las construcciones con ka y lo, el núcleo de la frase posesiva es el poseído y el poseedor funciona como modificador genitivo de éste. Pero en las construcciones con ko, el orden de las palabras es distinto: el poseedor generalmente se ubica después del nombre poseído. Incluso, el nombre poseedor puede aparecer precedido del subordinante to. De esta manera, se afirma que el poseído es 'la pertenencia del poseedor'.

(18) (a) $\mathrm{Y}$ (to) $\mathrm{X}$ prefijo-ko ( $\mathrm{Y}$ es nombre alienable)

portero to i-tiłame jawes [to eskwela la-ko] portero SUB 3-conservar llaves SUB escuela 3POS-CL 'El portero conserva las llaves de las escuela' (Lit: portero que conserva (las) llaves [que (son) pertenencia de la escuela]

(b) prefijo-Y (to) X prefijo-ko ( $\mathrm{Y}$ es nombre inalienable)

notsas $\emptyset$ - $\mathrm{t}^{\mathrm{e}} \mathrm{ek}^{\mathrm{w}} \mathrm{e}$ lax ${ }^{\mathrm{w}} \mathrm{ul}$ maestalu la-ko niños 3-buscar flauta maestra 3POS-CL 'Los niños están buscando la flauta de la maestra' (Lit: niños buscan flauta pertenencia de (la) maestra)

(c) $\mathrm{X}$ prefijo-Y (to) prefijo-ko (Y es nombre inalienable) ułam u-sikaje witji la-wet-es la-ko 1PRO/sG 1sG-visitar wichí/gente 3POS-lugar-PL 3POS-CL 'Yo visité el lugar (pertenencia) de los wichí'

En las construcciones que hemos registrado con ko el poseedor es siempre [+humano] y el poseído [-humano, -animado]. No obstante, $k o$ puede ser usado en construcciones posesivas con poseedor [-humano] únicamente, si el nombre poseedor se refiere a algún tipo de institución como por ejemplo, una escuela o un instituto: liblu eskwela ko 'los libros (pertenencia) de la escuela'; sijas instituto to la-ko 'las sillas (pertenencia) del instituto'.

Ko es usado en construcciones predicativas de pertenencia que expresan una noción de posesión permanente, en el sentido de que el ítem poseído es propiedad del poseedor. 
Considerando estos datos podemos pensar en una escala de posesión en base a dos parámetros control y proximidad y/o relación. ${ }^{17}$ Las construcciones posesivas con ko expresan una posesión permanente en el sentido de que el poseedor siempre está relacionado, de alguna forma, con el ítem poseído. El poseedor tiene por lo tanto, mayor control y proximidad sobre el ítem poseído y de esta manera es posible afirmar que dicho ítem le pertenece. Las construcciones con ka pueden indicar una posesión permanente, temporal o física, según el mayor o menor control que el poseedor tenga del ítem poseído y según la mayor o menor proximidad o relación que exista o se observe entre el poseedor y el poseído.

CUADRO 7

Escala de posesión

\begin{tabular}{|c|c|}
\hline $\begin{array}{l}\text { Parámetros: [control] y } \\
\text { [proximidad] y/o relación] }\end{array}$ & Construcciones posesivas con ko, lo y ka \\
\hline $\begin{array}{l}{[+ \text { control }]} \\
{[+ \text { proximidad }] \text { y/o }} \\
{[+ \text { relación }]}\end{array}$ & $\begin{array}{l}\text { Ko: } \mathbf{Y} \text { es de } \mathbf{X} \text {, porque } \mathrm{X} \text { siempre está con } \mathrm{Y} \text {; por lo tanto, } \\
\text { es posible afirmar que } \mathbf{Y} \text { es pertenencia de } \mathbf{X} \text {. } \\
\text { Lo: } \mathbf{Y} \text { es de } \mathbf{X} \text { porque } X \text { lo domestica; por lo tanto, es posible } \\
\text { señalar que } \mathbf{Y} \text { es la mascota de } \mathbf{X} \text {. } \\
\text { (Posesión permanente) }\end{array}$ \\
\hline $\begin{array}{l}\text { [+/-control] } \\
{[+/ \text {-proximidad }] \text { y/o }} \\
{[+/ \text {-relación }]}\end{array}$ & $\begin{array}{l}\text { Ka: Y puede o no ser de } \mathrm{X} \text {; se dice que } \mathrm{Y} \text { es de } \mathrm{X} \text { porque } \\
\text { es posible ver que } \mathrm{X} \text { usa a } \mathrm{Y} \text {, aparece o está con } \mathrm{Y} \text { (a veces o } \\
\text { siempre) pero no se afirma que } \mathbf{Y} \text { pertenece a } \mathbf{X} \text {. } \\
\text { (Posesión permanente, momentánea y física) }\end{array}$ \\
\hline
\end{tabular}

De esta manera una frase como $u$-ka hele 'mi yica' puede expresar posesión permanente (porque siempre tengo la yica y la uso, independientemente de que me pertenezca o no) o posesión temporal (aunque yo no sea la dueña, la yica es mía en el momento en que la uso). Por otra parte, una frase como $u-k a$ sija 'mi silla' sólo puede expresar posesión temporal y física: uso la silla en el momento pero no me pertenece. ${ }^{18}$

Ahora bien, una frase como hele to u-t ita la-ko 'la yica (pertenencia) de mi hermana' expresa posesión permanente: la yica pertenece a mi hermana porque mi hermana siempre la tiene y la lleva a donde va. Por el contrario, una frase como *sija to ułam la-ko no es posible, porque sólo puedo usar la silla cuando estoy presente en el lugar donde se encuentra la silla. El uso de $k o$ con silla es posible únicamente en la siguiente frase sija Instituto la-ko 'la silla pertenece al instituto’ porque la silla siempre está en el instituto. 
Esto nos permite explicar porqué ka y ko no siempre pueden ser usados en los mismos contextos como señalamos en el cuadro 5. Tomemos como ejemplo construcciones posesivas con los nombres hunat 'tierra' e inot 'agua'.

En (19) ambas construcciones expresan posesión permanente y es posible usar ambos clasificadores: en (a) la tierra es mía, porque yo trabajo en ella y la uso siempre. De igual manera en (b) la tierra pertenece a los wichí porque trabajan en ella y la usan siempre.

(19) (a) u-ka hunat 1POS/ sG tierra

'Mi jardín’ Lit: Mi tierra ${ }^{19}$

(b) hap suwele ihi hunat witfi $\emptyset$-ko FOC criollo estar tierra wichí 3POS-CL

'Los criollos están en la tierra (pertenencia) de los wichís'

Ahora bien, la frase en (20) expresa una posesión temporal porque se refiere al agua que él o ella recoge para usar (cocinar, beber, hervir); por ello, la construcción con ko no es posible ya que la relación entre el poseedor y el agua no es sólo momentánea.

(20) la-ka inot

3POS agua

'Su agua'

*inot to łam la-ko

\section{Conclusión}

En este trabajo hemos analizado los clasificadores de posesión $k a$, lo y ko observados en nuestro corpus. En primer lugar, hemos señalado sus particularidades y compatibilidades (cuadro 3). Luego, hemos analizado el comportamiento y uso de cada uno de ellos y planteamos como primera hipótesis que los clasificadores $k o, k a$ y lo indican tanto las características del ítem poseído como la relación de posesión que se establece entre ambos (cuadro 6). No obstante, las particularidades observadas respecto al uso de estos clasificadores (combinación con ciertos sufijos, distribución, nombres categorizados, etc.), en especial respecto de $k o$, plantean ciertas dificultades para situarlos en un determinado modelo o tipo de clasificadores. Finalmente, señalamos las nociones de posesión expresadas por las construcciones posesivas 
con ka, ko y lo. Este análisis nos ha permitido plantear como hipótesis una escala de posesión en base a dos parámetros control y proximidad y/o relación. Las construcciones posesivas con ko y lo expresan una posesión permanente en el sentido de que el poseedor siempre está relacionado, de alguna forma, con el ítem poseído (el poseído pertenece al poseedor, es propiedad del poseedor). Las construcciones con $k a$ pueden indicar una posesión permanente, temporal o física independientemente de que el poseído pertenezca o no al poseedor (cuadro 7).

\author{
Abreviaturas \\ CL: clasificador \\ POS: posesivo \\ SUB: subordinante \\ PL: plural \\ dem: demostrativo \\ 3: tercera persona \\ ( ): segmentos eliminados u omitidos
}

PL: plural

PRO: pronombre

VERB: verbalizador

SG: singular

1: primera persona

¿?: desconocimiento

* : formación no permitida en la lengua

\section{Notas}

* Deseamos agradecer a Jimena Terraza y a Marisa Censabella por sus observaciones y comentarios al respecto de este trabajo y a Sergio Merino por estar siempre dispuesto a enseñarnos su lengua.

${ }^{1}$ La abreviatura SM hace referencia a las letras iniciales del nombre y apellido del hablante con quien hemos trabajado.

${ }^{2}$ Información sobre el hablante SM: Edad: 42 años. Lugar y fecha de nacimiento: Ingeniero Juárez, 9/09/1968. Lugar de residencia: actualmente vive en Resistencia, periódicamente viaja a Ingeniero Juárez. Durante su infancia y juventud residió en varios lugares: Pozo Yacaré, El Sauzalito, Castelli y Salta. Lenguas: wichí y castellano.

${ }^{3}$ Por ejemplo, la lengua panare (lengua caribe) tiene veintiún clasificadores que categorizan a los nombres alienables en términos de su forma, estructura y consistencia. Así, el clasificador $u k u$ se usa con nombres referidos a líquidos como leche, sangre y café; el clasificador yiki, con nombres referidos a animales (incluso domésticos) y el clasificador kanowa, con nombres referidos a vehículos como canoa, camión, auto, etc. (AIKHENVALD, 2003, p. 128).

${ }^{4}$ Por ejemplo, en la lengua tariana (lengua arahuacana) que distingue posesión alienable e inalienable, los clasificadores de posesión pueden ser usados tanto con nombres alienables como inalienables. Así, el clasificador dapana 'casa' es usado 
con el nombre inalienable panisi 'hogar' y el clasificador ite 'animal', con el nombre alienable $t$ finu ‘perro’ (AIKHENVALD, 2003, p. 131).

${ }^{5}$ De acuerdo con Aikhenvald, la lengua dâw (lengua makú) es la única que tiene clasificadores para caracterizar al poseedor en términos de animacidad.

${ }^{6}$ Carlson y Payne utilizan el término función para indicar tanto 'el instrumento para hacer algo' como 'la finalidad del mismo'.

${ }^{7}$ El término relación individualizada usado por Carlson y Payne para referirse a ítems de posesión alienable, alude a relaciones o interacciones que se construyen en base a la utilidad o función que un ítem tiene para el poseedor; es decir, relaciones que están social o funcionalmente basadas.

${ }^{8}$ Por ejemplo, en manam (lengua austronesia) el nombre panana 'cabeza' es tratado como inalienable, en construcciones posesivas como panana-gu 'mi cabeza' (parte del cuerpo). O bien, como alienable en construcciones posesivas con diferentes clasificadores: panana iana-gu 'mi cabeza -cabeza del pez-' (posesión alimenticia); payana ne-gu 'mi cabeza -la que encontré-' (posesión general).

${ }^{9}$ De acuerdo con Heine existen tres tipos de construcciones posesivas: 1. las construcciones de posesión atributivas (Ej. My credit card 'mi tarjeta de crédito'); 2. las construcciones de posesión predicativa con el verbo tener Havecosntructions (Ej. I have a credit card ‘yo tengo una tarjeta de crédito’) y 3. las construcciones de posesión predicativa de pertenencia Belong-cosntructions (Ej. The car is Peter's 'el auto es de Pedro'; The car belongs to Peter 'el auto pertenece a Pedro').

Según el autor, la principal diferencia entre estas construcciones es semántica. Una construcción predicativa de pertenencia como The car belongs to Suzzane 'el auto pertenece a Susana’ está limitada a expresar una posesión permanente. Es decir, una relación en la que el ítem poseído es una propiedad del poseedor y que por lo tanto, el poseedor tiene derecho legal sobre el mismo. Una construcción predicativa con el verbo tener, como por ejemplo Suzzane has a car 'Susana tiene un auto', puede expresar no sólo una posesión permanente (Susana tiene un auto, esto es, le pertenece) sino también una posesión temporal. Es decir, una relación accidental o de control temporal en la que el poseedor puede disponer del ítem poseído pero por un tiempo limitado pero no puede reclamar derecho sobre el mismo (Susana tiene un auto pero no le pertenece). Una construcción de posesión atributiva como Suzzane's car 'el auto de Susana’ puede expresar no sólo posesión permanente o temporal sino también otros significados como por ejemplo: el auto que Susana quería pero nunca compró, el auto que vendió, compró, vió, mencionó, etc. La posesión atributiva denota posesión pero a la vez, admite significados que no tienen 
una relación de posesión discernible. No obstante, Heine sostiene que aunque las construcciones atributivas tienden a ser polisemánticas esto no implica que lo sean en todos sus usos. Según el contexto en que se usen pueden tener varias interpretaciones o una única interpretación, como por ejemplo Suzzane’s husband 'el esposo de Susana’ que denota, sin duda alguna, al hombre que está relacionado a Susana por lazo matrimonial.

${ }^{10}$ Los ejemplos han sido transcriptos tal como están citados en el texto de Heine.

${ }^{11}$ Heine distingue siete nociones de posesión en base a dos parámetros principales: control (que el poseedor tiene sobre el poseído) y tiempo (período de tiempo en que el poseído está ubicado en proximidad con el poseedor):

i. Posesión física (también denominada posesión momentánea) el poseedor y el poseído están asociados físicamente en el tiempo de referencia: Ej. a. I want to fill this form; do you have a pen? (posesión predicativa) b. Stewart, my glass is empty, can you bring me another one? (posesión atributiva).

ii. Posesión temporal (también denominada posesión accidental o control temporal) el poseedor puede disponer del poseedor por un tiempo limitado pero no puede reclamar derecho de propiedad sobre el poseído. Ej. a. I have a car that I use to go to the office, but it belongs to Judy (posesión predicativa) b. You can't have my car because it belongs to my wife (posesión atributiva).

iii. Posesión permanente (también denominada posesión inherente) el poseído es propiedad del poseedor, por lo cual el poseedor tiene título legal del poseído. Ej. a. Judy has a car, but I used all the time (posesión predicativa) b. My car has an accident, I have to buy a new one (posesión atributiva).

iv. Posesión inalienable el poseído se concibe como algo típicamente inseparable del poseedor. Ej. a. I have blue eyes / two sisters (posesión predicativa) b. My eyes / My sisters (posesión atributiva).

v. Posesión abstracta el poseído es un concepto invisible e intangible como una enfermedad o un sentimiento. Ej. a. He has no time / no mercy. (posesión predicativa) b. My thoughts (posesión atributiva).

vi. Posesión inalienable inanimada (frecuentemente referida como una relación parte-todo) el poseedor es inanimado. El poseído y el poseedor son considerados como inseparables. Ej: a. That tree has few branches (posesión predicativa) b. The ceiling of this room (posesión atributiva).

vii. Posesión alienable inanimada el poseedor es inanimado y el poseído es considerado como separable del poseedor. Ej. a. The tree has crows on it (posesión predicativa) b. The chairs from this room (posesión atributiva). 
${ }^{12}$ Hemos registrado sólo un ejemplo donde ko es prenominal:

ułam u-ko lawule-j

1PRO 1POS-CL cabello/hoja-PL

'Mis cabellos'

${ }^{13}$ De acuerdo con la información brindada por nuestro informante ka inot implica un "esfuerzo" por parte de las personas de ir a buscar y recoger el agua.

${ }^{14}$ En wichí estas relaciones de parentesco pueden expresarse también mediante los siguientes nombres inalienables: tos 'hijo', łose 'hija' y tfex ${ }^{w} a$ 'esposo/a'

${ }^{15}$ De acuerdo con Terraza (2009, p. 176-181), la derivación verbal en wichí es posible con dos raíces verbales semánticamente cercanas: -wu y jen- que se traducen por 'hacer'. Ambas exigen un morfema - $a$ al final del verbo derivado. Este morfema es denominado por la autora como un verbalizador:

wu-wej-a 'vestir' jen-t $\int$ emet-a 'trabajar'

hacer-ropa-VERB hacer-trabajo-VERB

${ }^{16}$ Este comentario es interesante porque en wichí las construcciones predicativas con tener emplean el verbo wen 'tener' que también significa 'ver'.

${ }^{17}$ La relación entre poseedor y el poseído puede referirse, como señalan Carlson y Payne, a distintos tipos: relaciones de parentesco, roles culturales, lugar de origen y residencia, propiedad individualizada genuina, etc.

${ }^{18}$ De acuerdo con la información brindada por nuestro informante la silla no puede ser mi propiedad porque no puedo trasladar y llevar la silla conmigo a todas partes.

19 ‘Mi jardín’ sería la parte de tierra que es mía, esto es, la parte de tierra que yo uso y trabajo. 


\section{Referencias}

AIKHENVALD, A. Classifiers a typology of noun categorization devices. Oxford: Oxford University Press, 2003.

CARLSON, R.; PAYNE, D. Genitive classifiers en Proceedings of the 4th Annual Pacific Linguistics Conference. Eugene: University of Oregon, p. 87-119, 1989.

GOLLUSCIO, L. Clases de sustantivos y sistema cultural: la posesión en wichí. Signo y Seña, Buenos Aires: Facultad de Filosofía y Letras Universidad de Buenos Aires, n. 3, 1993.

HEINE, B. Possession. Cognitive sources,forces and grammaticalization. Cambridge: Cambridge University Press, 1997.

TERRAZA, J. Gramática del wichí: fonología y morfosintaxis. Tesis (Doctorado) Universidad de Québec, Montreal, Canadá, 2009.

VIÑAS URQUIZA, M. T. Lengua Mataca, V. 2. Archivo de lenguas precolombinas. Buenos Aires: Centro de Estudios Lingüísticos, Universidad de Buenos Aires, 1974. 